\title{
Preoperative psychological adjustment and surgical outcome are determinants of psychosocial status after anterior temporal lobectomy
}

\author{
Bruce P Hermann, Allen R Wyler, Grant Somes
}

\begin{abstract}
This investigation evaluated the role of preoperative psychological adjustment, degree of postoperative seizure reduction, and other relevant variables (age, education, IQ, age at onset of epilepsy, laterality of resection) in determining emotional psychosocial outcome following anterior temporal lobectomy. Ninety seven patients with complex partial seizures of temporal lobe origin were administered the Minnesota Multiphasic Personality Inventory (MMPI), Washington Psychosocial Seizure Inventory (WPSI), and the General Health Questionnaire (GHQ) both before and six to eight months after anterior temporal lobectomy. The data were subjected to a nonparametric rank sum technique (O'Brien's procedure) which combined the test scores to form a single outcome index (TOTAL PSYCHOSOCIAL OUTCOME) that was analysed by multiple regression procedures. Results indicated that the most powerful predictors of patients' overall postoperative psychosocial outcome were: 1) The adequacy of their preoperative psychosocial adjustment, and 2) $A$ totally seizure-free outcome. Additional analyses were carried out separately on the MMPI, WPSI, and GHQ to determine whether findings varied as a function of the specific outcome measure. These results were related to the larger literature concerned with the psychological outcome of anterior temporal lobectomy.
\end{abstract}

EpiCare Center, Baptist Memorial Hospital, Memphis, Tennessee

and Departments of Neurosurgery, Psychiatry and Biostatistics, * University of Tennessee, Memphis, Tennessee

Semmes-Murphey Clinic, Memphis, Tennessee, USA

B P Hermann

A R Wyler

G Somes ${ }^{\star}$

Correspondence to: Dr Hermann, EpiCare Center, Baptist Memorial Center, Baptist Memorial Hospital, 910 Madison Avenue, Memphis

TN 38103, USA. and in revised form 28 August 1991.

Accepted 14 October 1991

The beneficial effects of anterior temporal lobectomy for the treatment of temporal lobe epilepsy have been demonstrated. ${ }^{3}$ The effects of surgery on patients' subsequent quality of life are less clear and the recent NIH Consensus Conference on Surgery for Epilepsy specifically noted that additional research was needed to clarify the psychological, social, and health status benefits of epilepsy surgery. ${ }^{25}$ Quality of life is a term that encompasses many aspects of life (for example, vocational, social, recreational, marital) and several groups have previously addressed some of these areas of postoperative status. ${ }^{610-1216-18212224}$ Another important dimension of quality of life concerns the patients' postoperative emotional and psychosocial adjustment.

This investigation used multiple measures of emotional and psychosocial status in a large sample of patients who had anterior temporal lobectomy to test the following hypotheses: 1) Patients with a totally seizure-free outcome would exhibit significantly greater improvements in self-reported emotional/psychosocial outcome compared with patients who had an excellent surgical outcome but were, nevertheless, not seizure-free; 2) The adequacy of patients' preoperative psychological and psychosocial status would be a significant predictor of their post-operative emotional and psychosocial status. Each of these points will be discussed.

First, improvements in psychiatric and social status have been found to be related to the degree of seizure relief provided by anterior temporal lobectomy. ${ }^{16}$ More recent findings have suggested that a complete cessation of seizure activity is most closely associated with major improvements in emotional and behavioural function. ${ }^{89}$ At first glance these findings may seem somewhat counterintuitive because it might expect that patients who received good surgical outcome (for example, $>80 \%$ or $90 \%$ reduction in seizure frequency) to exhibit good functional outcome. If, however, sharply improved emotional and psychosocial status is significantly dependent on total cure, then such findings might influence patient selection criteria, consideration for reoperation, or underscore the need for clinical trials to determine the most effective surgical procedure. To address the impact of surgical outcome on subsequent quality of life we compared patients who had excellent surgical outcome ( $>75 \%$ reduction), but who were not totally seizure-free, with a completely seizure-free group.

Second, Taylor ${ }^{23}$ has argued that the psychological/psychosocial outcome of epilepsy surgery cannot be accurately ascertained unless there is an understanding of the input, or the preoperative psychological status of the patient. There has been virtually no empirical investigation of this hypothesis. A clearer understanding of the relationship between patients' pre- and postsurgical psychological status has implications for prognoses regarding postoperative quality of life gains, and might suggest when in the natural history of a patient's epilepsy that surgical intervention would have the optimum effect on subsequent quality of life. Patients who present for surgical consideration with a history of longstanding psychological and social disability may remain functionally disabled regardless of surgical outcome, and therefore surgical consideration might be most appropriate if initiated before intractable epilepsy has exerted its deleterious 
neurological and social effects. This investigation examined patients' postoperative adjustment in the light of their preoperative status.

Research in this area is complicated by the fact that there is uncertainty as to both the best way to assess emotional and psychosocial status and the optimal method to analyse such data. Emotional/psychosocial adjustment can, and has been, assessed in a diversity of ways, for instance, through traditional psychiatric diagnostic categories (that is, DSMIII), family report, structured interview procedures, and standardised self report measures. ${ }^{46131419202223}$ Each of these methods has its own unique methodological strengths and weaknesses. Patient self-report is a widely used assessment method and one that we rely on to assess patient outcome. Within the self-report framework a variety of possible self-report measures could be used to assess functional outcome. Some of these instruments were designed specifically to assess patients with epilepsy, ${ }^{2}$ but most were not. Further, some tests/inventories are used almost exclusively in the USA (for example, the MMPI) ${ }^{7}$ while others are more widely used in the UK (for example, the General Health Questionnaire). 5

As anterior temporal lobectomy is performed worldwide it is uncertain whether findings regarding functional outcome might vary among surgical centres solely because of the different psychological tests used. Three common psychological tests therefore were used to examine the reproducibility of findings across different measures of behavioural/psychosocial outcome. A special statistical procedure was used that controls for the problems posed by the use of multiple outcome measures, and which results in derivation of a single index of psychosocial status despite the use of several tests. Combination of individual test scores into a unitary index of outcome offers several conceptual advantages, and may yield a more reliable and replicable index of outcome than reliance on individual psychometric tests.

\section{Method}

Subjects

Subjects consisted of a consecutive series of nonretarded (WAIS-R Full Scale IQ > 69), left hemisphere dominant patients who had anterior temporal lobectomy for treatment of intractable seizures of temporal lobe origin. Table 1 presents subjects in terms of their

Table 1 Subject Characteristics

\begin{tabular}{|c|c|c|}
\hline & Seizure-free $(N=53)$ & Significantly improved $(N=44)$ \\
\hline $\begin{array}{l}\text { Age (years) } \\
\text { Age at onset (years)(1) } \\
\text { Gender } \\
\text { Education (years) } \\
\text { WAIS-R Full Scale IQ } \\
\text { Resection (2) } \\
\text { Mean test-retest interval (mos) }\end{array}$ & $\begin{array}{l}30 \cdot 6(7 \cdot 9)^{\star} \\
10 \cdot 3(8 \cdot 96) \\
32 \mathrm{~F}, 21 \mathrm{M} \\
12 \cdot 97(2 \cdot 4) \\
90 \cdot 6(13 \cdot 1) \\
17 \text { left temporal } \\
36 \text { right temporal } \\
7.5\end{array}$ & $\begin{array}{l}30 \cdot 4(10 \cdot 8) \\
15 \cdot 0(11 \cdot 2) \\
25 \mathrm{~F}, 19 \mathrm{M} \\
12.9(2 \cdot 4) \\
92 \cdot 0(10 \cdot 8) \\
25 \text { left temporal } \\
19 \text { right temporal } \\
7.8\end{array}$ \\
\hline
\end{tabular}

surgical outcome. Patients who were rendered completely seizure free had an earlier age at onset and a greater proportion of patients with right temporal lobe epilepsy. There were no differences between the groups in age, gender, educational level, or WAIS-R Full Scale IQ, or time to follow up.

Preoperatively, all patients had long term video/EEG monitoring of spontaneous seizures with scalp electrodes and, in most cases, repeat monitoring with implanted subdural strip electrodes to confirm consistent unilateral temporal lobe origin of the seizures. Patients with MRI verified lesions (except for mesial temporal sclerosis) were excluded from the investigation. Such patients constituted a distinct minority (13\%) of our series of anterior temporal lobectomies and were heterogeneous in terms of the nature, location and volume of their lesion. Attention was therefore concentrated on the predominant patient group in our series, that is, those with chronic, intractable epilepsy not attributable to a mass lesion. Cerebral dominance for speech was determined by bilateral intracarotid sodium amytal testing. ${ }^{1}$

\section{Procedures}

Three standardised tests were used to assess emotional and psychosocial adjustment, the Minnesota Multiphasic Personality Inventory (MMPI), ${ }^{7}$ the Washington Psychosocial Seizure Inventory (WPSI), ${ }^{2}$ and the General Health Questionnaire (GHQ). ${ }^{5}$ The MMPI is a well known and widely utilised standardised measure of personality and emotional function that has three validity scales and ten clinical scales (hypochondriasis, depression, hysteria, psychopathic deviate, masculinity/femininity, paranoia, psychasthenia, schizophrenia, hypomania, and social introversion). ${ }^{7}$ The WPSI was developed specifically to assess numerous psychological and social issues and concerns of patients with epilepsy. It has three validity scales and eight clinical scales (family background, emotional adjustment, interpersonal adjustment, vocational adjustment, financial status, adjustment to seizures, medicine and medical management, overall psychosocial adjustment). ${ }^{2}$ Finally, the GHQ is a standardised measure of emotional status and was devised to identify individuals with a nonpsychotic psychiatric illness by assessing the severity of their psychiatric symptoms. ${ }^{5}$

The behavioural measures were administered preoperatively and six to eight months postoperatively. Surgical outcome at readministration of the behavioural measures was determined by ARW who was "blind" to the results of the psychological tests.

\section{Surgery}

There was generally about a $4.5 \mathrm{~cm}$ resection of lateral cortex from the nondominant temporal lobe, and $4.0 \mathrm{~cm}$ resection from the dominant temporal lobe. This was measured from the temporal tip (at the sphenoid wing) along the mid-temporal gyrus. Electrocorticography was not used to modify the amount of lateral cortex removed, and in fact has been 
abandoned on all patients monitored preoperatively with subdural strip electrodes. In all cases the hippocampus was resected and the amygdala was partially resected. First, lateral temporal cortex was removed medially to the level of the temporal horn of the lateral ventricle. Then, mesial temporal structures were removed posteriorly to the middle of the cerebral peduncle. In all cases the Cavitron ultrasound aspirator was used for brain dissection. The operating microscope was used by the surgeon in all procedures.

\section{Data analyses}

A methodological difficulty concerns the substantial number of dependent measures (scale scores) involved in outcome evaluations of this type. Several statistical procedures exist for evaluation of outcome when multiple endpoints (or dependent measures) are used (for example, Hotelling T, Bonferroni adjustments for multiple comparisons). While these approaches have advantages, they also have considerable limitations. ${ }^{15}$

Conceptual problems also exist with a series of univariate analyses. If several univariate analyses were carried out between seizure-free and significantly improved groups, what would it mean if a third or a half of the variables yielded between group differences? What would it tell us about the overall outcome, particularly when the dependent measures are known not to be factorially pure?

To address these and other problems, O'Brien devised a nonparametric rank sum test which is particularly appropriate for addressing treatment effects when multiple measures of outcome are utilised. ${ }^{15}$ The advantages of the O'Brien procedure are: 1) It provides a single overall probability statement on treatment outcome despite the use of multiple dependent measures; 2) It eliminates the statistical and conceptual problems associated with multiple comparisons, and, 3) As a rank test, it downgrades the effects of outliers.

The O'Brien procedure, as applied to this investigation, was carried out as follows. All 97 subjects were assigned a rank (from low to high, or from 1 to 97) based on their scale score for each and every dependent measure obtained preoperatively. That is, all subjects were first ranked from 1 to 97 on the MMPI Hs scale, then they were ranked on the D scale, and so on. Ranking was carried out for all eight clinical scales of the WPSI, the total GHQ score, and MMPI scales F, Hs, D, Hy, Pd, Pa, $\mathrm{Pt}, \mathrm{Sc}$, and $\mathrm{Ma}$. Each subject therefore received 18 preoperative rank scores (a rank for each of the 18 dependent measures). Each

Table 2 Results

\begin{tabular}{llll}
\hline Variable & $R$ & $R 2$ & Significant Predictor \\
\hline $\begin{array}{l}\text { TOTAL PSYCHOSOCIAL } \\
\text { ADJUSTMENT } \\
\text { MMPI }\end{array}$ & 0.60 & 0.36 & $\begin{array}{l}\text { Preoperative adjustment }(p=0.0001) \\
\text { Totally seizure free }(p=0.0009)\end{array}$ \\
GHQ & 0.52 & 0.27 & $\begin{array}{l}\text { Preoperative adjustment }(p=0.0001) \\
\text { Totally seizure free }(p=0.0008)\end{array}$ \\
WPSI & 0.40 & 0.16 & $\begin{array}{l}\text { Preoperative adjustment }(p=0.007) \\
\text { Age at onset }(p=0.01) \\
\text { Preoperative adjustment }(p=0.0001)\end{array}$ \\
\hline
\end{tabular}

subject's 18 preoperative ranks were then summed across scales and this value served as his/her preoperative score. This procedure was then repeated for the postoperative tests. A higher sum of ranks was indicative of increased psychopathology and poorer psychosocial function.

These pre- and postoperative sum of rank scores were then analysed by multiple regression procedures. The criterion measure was the postoperative sum of ranks score while the predictor variables were: 1) The subjects' preoperative sum of ranks scores; 2) Surgical outcome (totally seizure free, significantly improved); 3) Years of education; 4) Chronological age; 5) WAIS-R Full Scale IQ; 6) Laterality of temporal lobe resection (left, right temporal lobe); and 7) Age at onset of epilepsy.

The primary outcome (dependent) measure involved the sum of ranks using all psychological tests combined (MMPI, WPSI, GHQ) and is called TOTAL PSYCHOSOCIAL ADJUSTMENT. To determine the consistency and reproducibility of findings across the individual psychometric tests, the analyses were repeated for each of the three tests (MMPI, WPSI, GHQ) separately.

Stepwise multiple regression was utilised to control for the collinearity that existed among the predictor variables. Hence, variables which were identified as significant predictors of postoperative psychological status were independent predictors adjusted for other variables in the model. Because several multiple regression analyses were computed (each incorporating seven predictor variables) a p-value of $<0.01$ was used to identify significant predictor variables to minimise Type 1 error.

\section{Results}

TOTAL PSYCHOSOCIAL ADJUSTMENT

For this measure, the primary outcome index, table 2 shows that the multiple $R$ was 0.60 indicating that $36 \%$ of the variance was accounted for by the predictor variables. Two predictor variables reached statistical significance. The most powerful predictor of postoperative adjustment was the patients' preoperative adjustment $(p=0.0001)$. A totally seizure free outcome was also significantly associated with improved postoperative adjustment $(p=0.0009)$.

\section{Individual psychological tests}

For the MMPI the multiple $R$ was 0.52 indicating that $27 \%$ of the variance was accounted for by the predictor variables (table 2). Two variables were significant predictors of postoperative adjustment. Preoperative psychological adjustment was a direct predictor of postoperative adjustment $(p=0.0001)$, and a totally seizure free outcome was significantly associated with better postoperative adjustment $(p=0.0008)$.

For the GHQ the multiple $R$ was 0.40 indicating that the predictor variables accounted for $16 \%$ of the variance. Better preoperative adjustment was associated with good 
postoperative adjustment $(p=0.007)$ as was a later age at onset of epilepsy $(p=0.01)$.

Finally, for the WPSI the multiple $R$ was 0.62 indicating that $38 \%$ of the variance was accounted for by the predictor variables. Only one variable was predictive of postoperative adjustment, that is, preoperative adjustment $(p=0.0001)$. Surgical outcome was marginally significant $(p=0.03)$.

\section{Discussion}

First to be overviewed will be the results derived from the composite behavioural/psychosocial index (TOTAL PSYCHOSOCIAL ADJUSTMENT) followed by a discussion of the individual MMPI, WPSI, and GHQ findings. These results will be related to the hypotheses specified in the introduction. The limitations of this investigation will then be reviewed.

\section{Preoperative psychological status as a predictor of} postoperative status

The major dependent variable of interest was TOTAL PSYCHOSOCIAL ADJUSTMENT. This was a composite measure of behavioural and psychosocial outcome, reflecting the combined rank sum of scores on the MMPI, WPSI, and GHQ. Analysis of TOTAL PSYCHOSOCIAL ADJUSTMENT revealed that of the potential predictor variables, the strongest predictor of the adequacy of the patients' postoperative psychological adjustment was the adequacy of their preoperative adjustment. Patients who exhibited poor (or good) emotional and psychosocial function preoperatively continued to exhibit poor (or good) emotional and psychosocial function postoperatively. Strong support is therefore provided to Taylor's hypothesis that evaluation of psychosocial outcome cannot meaningfully proceed without knowledge of the patients' psychosocial condition before surgery. ${ }^{23}$ This consistency in preversus postoperative behaviour is in some agreement with other findings as well. For instance, Taylor and Falconer ${ }^{24}$ found a significant correlation $(r=0.60, p<0.001)$ between pre- and postoperative social adjustment scores, with the patients' preoperative mental status (normal, neurotic, psychopathic) serving as significant predictors of postoperative social adjustment. In general, good preoperative mental status (normal) predicted good postoperative social adjustment, while poor postoperative social adjustment was predicted by poor preoperative mental status (psychosis, psychopathic).

While changes in psychological and social functioning following anterior temporal lobectomy have been of considerable interest, the degree of consistency in behaviour from pre- to postoperative assessments is worth noting. This consistency in behaviour may partly help account for those patients who experience good surgical outcome but who are found to exhibit poor postoperative social adjustment. Such patients have always been relatively difficult to account for and hypotheses regarding their poor functional outcome have often centred on the nature of their preoperative personality. ${ }^{4117}$ Years of poor social and psychological adjustment simply may not be overcome by the sudden relief of seizures. Anterior temporal lobectomy has traditionally been viewed as a final treatment option, considered only after years of medication trials and significant exposure to the deleterious psychological and social effects of poorly controlled epilepsy. The current results raise the hypothesis that to maximise postoperative functional status, surgery might reasonably be considered sooner than is typically the case, before the organic, behavioural and psychosocial sequellae of chronic epilepsy have created relatively resistant psychological and social difficulties.

It would be of interest to determine whether preoperative status is a major predictor of postoperative outcome in other quality of life areas (for example, independent living, social interaction). If that were the case, then it would suggest the need for directed and comprehensive postoperative rehabilitation efforts for those who come to surgery with significant social, vocational, and interpersonal problems. Surgery might then be viewed as one component of a comprehensive intervention and rehabilitation effort.

Surgical outcome as a predictor of postoperative status

The other variable which was a significant predictor of TOTAL PSYCHOSOCIAL ADJUSTMENT was whether or not the patient experienced complete seizure control. This replicates our earlier report that sharply improved behavioural/emotional status was associated with a totally seizure-free outcome $^{89}$ and is perhaps a more rigorous test of this hypothesis because premorbid psychological status was controlled statistically in this investigation. Rausch and Crandall $^{16}$ first reported that an essentially seizure free outcome was associated with gains in psychosocial outcome following anterior temporal lobectomy, whereas patients who were significantly improved (that is, $>75 \%$ reduction in seizure frequency) were essentially unchanged relative to preoperative status. In our outcome rating system the occurrence of any seizure activity (including auras) following anterior temporal lobectomy relegated a patient to the nonseizure-free outcome category. Our significantly improved group therefore consisted of patients who experienced at least a $75 \%$ reduction of seizure frequency, patients with auras (simple partial seizures) only, patients who experienced a seizure only when they unilaterally reduced their medications, and other examples of excellent but not totally seizure-free outcomes. Even with the addition of these patients with good surgical outcomes to the non-seizure-free group, patients who experienced a complete cessation of seizure activity still showed a more positive psychological outcome attesting to the strength of the effect.

It should be noted that preoperative psychological status and seizure-free outcome, while 
highly statistically significant, accounted for a moderate amount of the variance in TOTAL PSYCHOSOCIAL ADJUSTMENT (36\%) indicating that other, as of yet unidentified variables account for the majority of the remaining variance in behavioural/psychosocial outcome. Several reasonable potential predictor variables (that is, $I Q$, age, age at onset of epilepsy, education, laterality of temporal lobectomy) failed to predict psychosocial outcome suggesting that other factors need to be added to this model in the future.

Consistency of findings across individual tests Another interest of this investigation concerned the reproducibility of outcome findings across three conventional psychological tests, the MMPI, WPSI, and GHQ. With interest growing in the quality of life following anterior temporal lobectomy, it is likely that research in this area will expand. Temporal lobectomy is conducted at epilepsy centres throughout the world, and there is variability across centres as to how behavioural and psychosocial outcome is assessed. Thus it is important to determine the consistency in findings that would be obtained within and between samples of patients when several different but representative psychological tests are used. The results revealed some significant variability in findings as a function of the specific psychological test used. The similarities and differences can be seen by examining the predictors for each test in table 2. Across tests, however, the most consistent predictor of postoperative psychological status (significant for all three tests) was the adequacy of the patients' preoperative psychological status. Surgical outcome was significant for one (MMPI), and marginally significant for a second (WPSI) of the three tests. Later age at onset of epilepsy was associated with improved psychosocial outcome on the GHQ, but not on the MMPI or WPSI. The important implication of this variability between tests is that different centres may obtain variability in results when addressing the same issue solely because of the use of different outcome measures.

Combining individual psychological measures into a single index (TOTAL PSYCHOSOCIAL ADJUSTMENT) is uncommon when dealing with tests of emotional and psychosocial function, but has long been the case in other areas of assessment (for example, WAIS Full Scale IQ). Such an approach has both strengths and limitations, and has been discussed at length (with little apparent consensus) in the health services research literature. The current results support Taylor's suggestion that a single index approach has utility in the epilepsy surgery literature, and further investigation in this regard appears warranted.

There are several limitations inherent in this investigation, the most prominent of which is our total reliance on self-report measures. Selfreport measures constitute only one way to assess psychosocial outcome. Confidence in the effects of preoperative psychological status and seizure-free outcome need to be evaluated with other outcome assessment techniques to determine the convergent validity of these findings beyond the self-report format. Investigation and confirmation from other sources (for example, clinician ratings, reports from family measures) are needed.

Another limitation was the relatively short duration of follow up-six to eight months. While this period of follow up is too short to be of long-term predictive value, it is relevant to note that these findings are similar to those reported in series where the follow up was considerably longer. ${ }^{1624}$ Nevertheless, longer follow up is required to determine the stability of these findings. We are currently assessing our own sample at five years post-operatively, but those results will not be available for some time and the generalisability of the current findings to other outcome intervals is needed. Additionally, some seizure free patients will experience reoccurrence of seizures. Longitudinal investigations will be needed to investigate the effects of changes in seizure status on psychosocial functioning.

In conclusion, the adequacy of preoperative psychosocial status as well as surgical outcome are significant determinants of postoperative psychosocial adjustment. These variables need to be investigated in other areas of outcome (such as, vocational status, dependent/independent living status) to determine the general effects. The results of such investigations are likely to enhance our understanding of the determinants of quality of life following surgery, and lead to an understanding of the optimal timing of surgical intervention to maximise the probability of improving patients' quality of life.

1 Blume WT, Grabow JD, Darley FL, et al. Intracarotid amobarbital test of language and memory before temporal lobectomy for seizure control. Neurology 1973;23: 812-9.

2 Dodrill CB, Batzel LW, Queisser HR, Temkin NR. An objective method for the assessment of psychological and social problems among epileptics. Epilepsia 1980;21: 123-35.

3 Engel J Jr., ed. Surgical treatment of the epilepsies. New York: Raven Press, 1987

4 Ferguson SM, Rayport M. The adjustment to living without epilepsy. F Nerv Ment Dis 1965;140:26-37.

5 Goldberg $\mathrm{D}$. The detection of psychiatric illness by questionnaire. Loldberg D. The detection of psychiatric illness

6 Green JR, Steelman HF, Duisberg REH, McGrath WB, Wick SH. Behavioural changes following radical temporal lobe excision in the treatment of focal epilepsy, in Association for Research in Nervous and Mental Disease. The brain and human behaviour, vol 36). Baltimore: Williams and Wilkins, 1958: 295-315.

7 Hathaway SR, McKinley JC. The MMPI manual. New York: Psychological Corporation, 1967.

8 Hermann BP, Wyler AR. Depression, locus of control, and the effects of epilepsy surgery. Epilepsia 1989;30:332-8.

9 Hermann BP, Wyler AR, Ackermann B, Rosenthal T. Shortterm psychological outcome of anterior temporal lobectomy. F Neurosurg 1989;71:327-34.

10 Hill D, Pond DA, Mitchell W, Falconer MA. Personality changes following temporal lobectomy for epilepsy. $\mathcal{F}$ Ment Sci 1957;103:18-27.

11 Horowitz MJ, Cohen FM. Temporal lobe epilepsy: effect of lobectomy on psychological functioning. Epilepsia lobectomy on

12 Jensen I. Temporal lobe epilepsy: social conditions and rehabilitation after surgery. Acta Neurol Scand 1976; rehabilitation

13 Jensen I, Larsen JR. Mental aspects of temporal lobe epilepsy: follow up of 74 patients after resection of a temporal lobe. $f$ Neurol Neurosurg Psychiatry 1979;42:256-65.

14 Koch-Weser M, Garron DC, Gilley DW, et al. Prevalence of psychological disorders after surgical treatment of epilepsy. Arch Neurol 1988;45:1308-11.

15 O'Brien P. Procedures for comparing samples with multiple 
endpoints. Biometrics 1984;40:1079-87.

16 Rausch R, Crandall PH. Psychological status related to surgical control of temporal lobe seizures. Epilepsia 1982;23:191-202.

17 Rausch R, McCreary C, Crandall PH. Psychosocial functioning following successful surgical relief from seizures. Evidence of prediction from preoperative personality characteristics. I Psychosom Res 1977;21:141-6.

18 Savard RJ, Walker E. Changes in social functioning after surgical treatment for temporal lobe epilepsy. Soc Work 1965;10:87-95.

19 Serafetinides EA. Aggressiveness in temporal lobe epileptics and its relation to cerebral dysfunction and environmental factors. Epilepsia 1965;6:33-42.

20 Serafetinides EA. Psychosocial aspects of neurosurgical management of epilepsy. In: Purpura DP, Penny JK, Walter RD, eds. Advances in neurology, vol 7 (Neurosurgical management of the epilepsies). New York: Raven Press, 1975.
21 Sherwin I. Psychiatric outcomes following surgery for temporal lobe epilepsy. In: Porter R, Mattson RH, Ward AA, Dam M, eds. Advances in epileptology: XVth international symposium. New York: Raven Press, 1984: 497-501

22 Simmel ML, Counts S. Clinical and psychological results of anterior temporal lobectomy in patients with psychomotor epilepsy. In: Baldwin M, Bailey P, eds. Temporal lobe epilepsy. Springfield: Charles C Thomas, 1958: 1247-61.

23 Taylor DC. Psychiatric and social issues in measuring input and output of epilepsy surgery. In: Engel I, ed. Surgical treatment of the epilepsies. New York: Raven Press, 1987: 485-503.

24 Taylor DC, Falconer MA. Clinical, socioeconomic, and psychological changes after temporal lobectomy for epipsychological changes after temporal
lepsy. Br $¥$ Psychol 1968;114:1247-61.

25 US Department of Health and Human Services. Surgery for epilepsy. NIH consensus development conference consensus statement. Washington DC: NIH, 1990. 